\title{
Deuterium Quadrupole Coupling Constants in Deuterochloroacetylene
}

\author{
N. Heineking, M. Andolfatto, U. Keussen, A. Mues, and H. Dreizler \\ Abteilung Chemische Physik im Institut für Physikalische Chemie \\ der Christian-Albrechts-Universität zu Kiel \\ Z. Naturforsch. 44a, $735-737$ (1989); received June 2, 1989 \\ The deuterium and chlorine nuclear quadrupole coupling constants have been determined for both \\ of the chlorine isotopomers of deuterochloroacetylene ( $\mathrm{DCCCl}$ ), along with the rotational, centrifugal \\ distortion, and chlorine spin-rotation coupling constants. The values are $B=5186.97631(22) \mathrm{MHz}$, \\ $D_{J}=1.13(2) \mathrm{kHz}, e Q q(\mathrm{Cl})=-79.7358(19) \mathrm{MHz}, c_{I}(\mathrm{Cl})=1.10(31) \mathrm{kHz}$, and $e Q q(\mathrm{D})=207.0(28) \mathrm{kHz}$ \\ for $\mathrm{DCCCl}-35$, and $B=5084.17839(30) \mathrm{MHz}, D_{J}=0.99(2) \mathrm{kHz}$, e $Q q(\mathrm{Cl})=-62.8451(27) \mathrm{MHz}$, \\ $c_{I}(\mathrm{Cl})=0.76(43) \mathrm{kHz}$, and $e Q q(\mathrm{D})=205.8(39) \mathrm{kHz}$ for DCCCl-37.
}

\section{Introduction}

Recently we reported on the deuterium quadrupole coupling constants of deuterohaloacetylenes [1] with the exception of deuterochloroacetylene. To fill the gap we now investigated the microwave spectra of the ${ }^{35} \mathrm{Cl}$ - and ${ }^{37} \mathrm{Cl}$-isotopomers of this molecule as well. We used our microwave Fourier transform (MWFT) spectrometers in X-band $(8.0-12.4 \mathrm{GHz})$ [2], K-Band $(18.0-26.4 \mathrm{GHz})$ [3], and V-band $(26.4-40 \mathrm{GHz})$ [4]. The high resolution of these instruments was essential for the investigations. A comparison of our results with the beam maser data of Tack and Kukolich [5] shows that static gas MWFT spectroscopy approaches the accuracy of molecular beam spectroscopy.

\section{Experimental}

Deuterochloroacetylene ( $\mathrm{DCCCl})$ was prepared by dehydrohalogenation of cis-1,2-dichloroethylene with excess molten KOD. The ratio of the yields of DCCCl and $\mathrm{HCCCl}$ was approximately $1: 3$ (as determined by microwave spectroscopy). The sample was stored at liquid $\mathrm{N}_{2}$ temperature in a dark place.

The recordings covered the rotational transitions $J=1-0, J=2-1$, and $J=3-2$, in the frequency range $8-33 \mathrm{GHz}$. We used the spectrometers men-

Reprint requests to Prof. H. Dreizler, Abt. Chemische Physik, Institut für Physikalische Chemie der Universität Kiel, Ludewig-Meyn-Str. 8, D-2300 Kiel, FRG. tioned above. The temperature of the sample was around $230 \mathrm{~K}$, and the pressure around $0.2 \mathrm{~Pa}$ (1-2 mTorr). The determination of the frequencies was performed by a fit procedure [6] using the time domain signals. The results are given in Tables 1 and 2 .

\section{Analysis}

For the analysis we used only resolved components of the multiplets of the rotational transitions $J=1-0$ through $3-2$. The procedure was similar to that given in [1] for DCCBr, and is based on the complete Hamiltonian including matrix elements off-diagonal in $J$ [7]. Our programme SYM2QS [8] was used. The results are enclosed in Tables 1 and 2. In Table 3 we compare our results with older data. Table 4 of [1], which summarizes the deuterium quadrupole coupling constants of the other haloacetylenes, should also be considered: Systematic trends with varying halogen mass cannot be ruled out, but the experimental accuracy so far achievable does not allow us to be more definitive.

\section{Acknowledgements}

We thank the members of our group for help and discussions. Funds provided by the Deutsche Forschungsgemeinschaft, the Fonds der Chemie, and the Land Schleswig-Holstein are gratefully acknowledged. 
Table 3. Comparison of the molecular parameters of $\mathrm{DCCCl}$ as determined in this work with earlier data.

\begin{tabular}{|c|c|c|c|c|}
\hline \multirow[t]{2}{*}{ Constant } & \multicolumn{2}{|l|}{ DCCCl-35 } & \multicolumn{2}{|l|}{$\mathrm{DCCCl}-37$} \\
\hline & this work & {$[5]$} & this work & [9] \\
\hline$(\mathrm{MHz})$ & $5186.97631(22)$ & - & $5084.17839(30)$ & \\
\hline$(\mathrm{MHz})$ & $0.00113(2)$ & - & $0.00099(2)$ & - \\
\hline$B-2 D_{J} \quad(\mathrm{MHz})$ & $5186.97405(22)$ & $5186.9739(10)$ & $5084.17641(30)$ & $5084.24(1)$ \\
\hline$e Q q(\mathrm{Cl})(\mathrm{MHz})$ & $-79.7358(19)$ & $-79.7395(10)$ & $-62.8451(27)$ & $-63.12(1)$ \\
\hline$c_{I}(\mathrm{Cl}) \quad(\mathrm{kHz})$ & $1.10(31)$ & $1.3(5)$ & $0.76(43)$ & - \\
\hline$e Q q(\mathrm{D})(\mathrm{kHz})$ & $207.0(28)$ & $208.5(15)$ & $205.8(39)$ & - \\
\hline
\end{tabular}

[1] N. Heineking, M. Andolfatto, C. Kruse, W. Eberstein, and H. Dreizler, Z. Naturforsch. 43 a, 755 (1988).

[2] G. Bestmann and H. Dreizler, Z. Naturforsch. 37 a, 58 (1982).

[3] P. Wolf and H. Mäder, Mol. Phys. 64, 43 (1988).

[4] Ch. Keussen, N. Heineking, and H. Dreizler, Z. Naturforsch. 44a, 215 (1989).

[5] L. M. Tack and S. G. Kukolich, J. Mol. Spectrosc. 94, 95 (1982).
[6] J. Haekel and H. Mäder, Z. Naturforsch. 43a, 203 (1988).

[7] W. Gordy and R. L. Cook, Microwave Molecular Spectra, $3^{\text {rd }}$ Ed., Chapter XV, John Wiley, New York 1984.

[8] Authors: B. Kleibömer and J. Gripp, Kiel.

[9] A. A. Westenberg, J. H. Goldstein, and E. B. Wilson jr., J. Chem. Phys. 17, 1319 (1949). 